\title{
Motivaciones de los estudiantes Universitarios ante la práctica de actividad físico-deportiva de tiempo libre. Las actividades náuticas
}

\author{
Antonio ALÍAS, José M. AGUILAR , Antonia I. HERNÁNDEZ-RODRÍGUEZ \\ Universidad de Almería, España
}

(Recibido 13 Marzo, 2016; Aceptado 18 Junio, 2016)

RESUMEN: El estudio de los perfiles motivacionales proporciona información detallada sobre los hábitos de los grupos de personas hacia la práctica de la actividad física, permitiendo poder fomentar una motivación más positiva y conseguir una mayor adherencia a la práctica. Así el objetivo de este estudio ha sido clarificar cuáles son las motivaciones frente a la práctica de actividad físico-deportiva de una muestra de jóvenes universitarios, incidiendo especialmente en las actividades náuticas. La muestra estuvo compuesta por 1011 estudiantes de la Universidad de Almería (España). El instrumento utilizado fue el cuestionario de hábitos físicos-deportivos y de práctica de deportes náuticos. Los resultados obtenidos apuntan que los principales motivos para desarrollar la práctica deportiva fueron la flexibilidad horaria y una adecuada a la disponibilidad de su tiempo libre, seguido de la cercanía a su domicilio de las instalaciones. Otros motivos son por diversión, o estar con su grupo de amigos. Respecto a las actividades náuticas, se decide practicar o no sólo porque les gustan, seguido del interés por estar en contacto con el medio natural y acuático. Las modalidades náuticas más practicadas son el piragüismo y la natación. En relación al abandono de la práctica deportiva, claramente se produce por la falta de tiempo por el estudio o el trabajo. Es evidente que la falta de tiempo por los estudios o por el trabajo, perjudica gravemente la adherencia a la actividad física, pero existen porcentajes muy altos de personas (62\%) que admiten no practicar por pereza y desgana, por lo que se deben buscar nuevas estrategias de motivación para que aumente la adherencia a la actividad físico deportiva.

Palabras clave: Motivación, práctica de actividad física y deportiva, actividades náuticas, hábitos físicos y deportivos, universitarios.

Motivations of the University students in the physical and sports practice of free time. The nautical activities

ABSTRACT: The study of motivational profiles provides detailed information on the habits of groups
of people to the practice of physical activity, allowing fostering a more positive motivation and
achieving greater adherence to practice. So the aim of this study was to clarify the motivations are
against the practice of physical and sporting a sample of university students, with special emphasis on
water sports activity. The sample consisted of 1011 students from the University of Almeria (Spain).
The instrument used was the questionnaire physical - sporting habits and water sports. The results
indicate that the main reasons for developing sport and flexible hours were adequate to the availability
of leisure, followed by proximity to your home facilities. Other reasons are for fun, or to be with his 
friends. Concerning water activities, it was decided not to practice or just because they like them, followed by the wish to be in contact with the natural aquatic environment. The most practiced nautical activities are canoeing and swimming. In relation to the abandonment of the sport is clearly caused by the lack of time for study or work. Clearly the lack of time for study or work, seriously undermines adherence to physical activity, but there are very high percentages (62 \%) who admit no laziness and unwillingness to practice, so you should seek new motivational strategies to increase adherence to physical activity sports.

Keywords: Motivation; practice of physical and sports activity; nautical activities; Physical and sports habits; university student.

${ }^{1}$ Correspondencia: José Manuel Aguilar Parra. Departamento de Psicología. Área de Psicología Evolutiva y de la Educación. Ctra. De Sacramento, s/n. 04120. La Cañada de San Urbano (Almería). E mail:jmaguilar@ual.es.

La importancia que tiene la motivación hacia la práctica de actividad físico-deportiva en estudiantes es un tema de actualidad que ha sido analizado desde diferentes perspectivas (Ames, 1995; Duda y Nicholls, 1992; Nicholls, 1984; Roberts y Treasure, 1995; entre otros). Tal y como señalan Almagro, Sáenz-López y Moreno-Murcia (2012) el estudio de los perfiles motivacionales está permitiendo determinar los diferentes patrones motivacionales existentes en una muestra de estudio concreta, atendiendo a diferentes variables motivacionales, con el objeto de poder proporcionar información detallada a los adultos significativos sobre las características particulares de su grupo y, con ello, poder fomentar una motivación más positiva y conseguir una mayor adherencia a la práctica.

Para tratar de explicar cuáles son las motivaciones en los estudiantes universitarios hacia la práctica deportiva, se han aplicado diferentes teorías motivacionales, siendo la teoría de la autodeterminación (TAD) (Deci y Ryan, 2000; Ryan y Deci, 2007) la que ha tratado de explicar las diferentes consecuencias del estado de motivación en las personas, entre ellas las consecuencias afectivas. Estas motivaciones necesitan de nuevos estudios para analizar y profundizar en los hábitos de práctica de actividades físico-deportivas de los sujetos en su tiempo libre, valorando especialmente las actividades náutica-recreativas y deportes náuticos, y determinado cuáles son sus motivaciones hacia dicha práctica. En este sentido, se considera que el éxito en la práctica de actividad físico-deportiva, depende en gran medida de los comportamientos hacia la práctica, o no, de actividad físico-deportiva por parte de las personas que están el entorno del joven (Delgado y Tercedor, 2002) y de la motivación de los estudiantes, debido a que la idea principal de la Teoría de las Metas de Logro consiste en que la persona es percibida como un organismo intencional, dirigido por unos objetivos hacia una meta que opera de forma racional (Nicholls, 1984). Así que resulta pertinente dotarnos de nuevos estudios que permitan a los profesionales conocer las motivaciones de los sujetos hacia la práctica de actividad físico-deportiva durante su tiempo libre (Martínez, Hellín, Pavón y Moreno-Murcia, 2010) y dotarnos de otros que permitan medir qué lugar ocupa la práctica de actividades náuticas-recreativas y deportes náuticos.

El Centro de Investigaciones Sociológicas (2005) ha arrojado mucha luz en el conocimiento de los comportamientos y las motivaciones de los españoles hacia la práctica de 
actividad físico-deportiva. Según García-Ferrando (2005), la creciente importancia de disponibilidad de tiempo libre en nuestra sociedad y el disfrute de éste, ha ido originando una mayor diversificación en las actividades físico-deportivas. La población española que practica algún tipo de deporte se sitúa en el $47 \%$ y entre ellos los deportes náuticos se sitúan por debajo del 1\% (García-Ferrando y Llopis, 2010). En el contexto universitario, Castillo y Jiménez (2011) constatan que un 41,9\% practicaban alguna clase de actividad físico-deportiva. GarcíaFerrando (2001), en los últimos veinticinco años, estableció un patrón de evaluación de los motivos que conducen a la práctica deportiva en España que, en gran medida, sigue vigente y que, sobretodo, ha sido refrendado por la investigación empírica. Las variables motivacionales que influyen dependen de la práctica deportiva y del tipo de deporte practicado. GarcíaFerrando y Llopis (2010) destacan por orden de importancia: diversión, pasar el tiempo libre y por encontrarse con los amigos, como los principales determinantes. En este sentido, el interés en la investigación sobre las motivaciones en la población de estudiantes universitarios persiste en encontrar una relación entre la práctica de actividad físico-deportiva y los motivos que fomentan el inicio, el mantenimiento o el abandono de dicha práctica. Pavón, Moreno, Gutiérrez y Sicilia (2003) mostraron los diferentes motivos existentes entre el estudiantado universitario de Murcia, Valencia y Almería. Así, para los estudiantes que practicaban las motivaciones se centraban en la competición, la capacidad personal y la aventura, mientras que los que no practicaban estaban más interesados por la salud. En esta misma línea, Ruiz, García y Hernández (2001), Pavón y Moreno-Murcia (2008) y Alonso y García (2010), entre otros, mostraron que entre los universitarios el motivo principal para no practicar actividades físicodeportivas es la falta de tiempo. Por lo tanto, se podría apuntar a la motivación como uno de los predictores de la práctica de actividad físico-deportiva entre los estudiantes. Wigfield y Eccles (2000) señalaron la idea que los motivos son conceptos dinámicos que varían en función del tiempo de práctica del ejecutante, con la finalidad de que el alumnado no abandone la práctica deportiva. Asimismo, la etapa universitaria es el momento apropiado para promocionar la práctica de actividad física-deportiva, pues en ella tiene lugar un porcentaje considerable de abandono de la practica por parte del estudiantado (Pavón y Moreno, 2006).

Por todo ello, el objetivo de este estudio fue clarificar cuáles son las motivaciones frente a la práctica de actividad físico-deportiva de una muestra de jóvenes universitarios, incidiendo especialmente en las actividades náuticas, por comprender una serie de actividades deportivas en auge sobre todo en zonas costeras, en este caso, la costa de Almería.

\section{Método}

\section{Participantes}

La muestra se ha extraído de la población estudiantil de la Universidad de Almería, con un tamaño muestral de 1.011 participantes. 463 Hombres $(45,8 \%)$ y 548 mujeres $(54,2)$ con una media de edad de 21,98 $(D T=4,68)$. El procedimiento de muestreo ha sido polietápico estratificado por facultades, con afijación proporcional en la primera fase y, posteriormente, con selección aleatoria por conglomerados de los grupos de clase, de cada uno de los cursos y grupos de los estudios existentes en cada facultad. 


\section{Instrumentos}

El instrumento utilizado ha sido "Cuestionario de hábitos físicos-deportivos y de práctica de deportes náuticos” diseñado ad hoc para esta investigación a través del cuestionario de García Ferrando “Encuesta de hábitos deportivos de los Españoles 2005”. El cuestionario fue autoadministrado informáticamente mediante plataforma virtual del campo andaluz. Para probar la calidad de los datos obtenidos, se evaluaron interjueces para posteriormente someter el cuestionario definitivo a un estudio piloto para evaluar distintos aspectos de lenguaje, sentido de las preguntas, fiabilidad de las respuestas, la forma de recoger los datos, el tiempo medio necesario para completar el cuestionario, los diferentes itinerarios y preguntas filtro, etc. Dicha validación se realizó aplicando el cuestionario a tres grupos (117 alumnos) de la Universidad de Almería, que no formaron parte de la muestra definitiva.

\section{Procedimiento}

Tras la elección de la muestra, se procedió a la pasación de los cuestionarios que se realizó a través de la aplicación Mr. Interview (plataforma Dimensions) que es una solución completa para la creación, captura y administración de encuestas en varios idiomas y por múltiples vías.

\section{Análisis de datos}

El análisis de datos se ha realizado mediante el programa estadístico SPSS 20. No obstante, como se trata de un estudio básicamente cualitativo, únicamente se ha trabajado con porcentajes de respuesta a través de estadísticos descriptivos complementados por Pruebas t y ANOVAS oneway valorando el tamaño del efecto de una variable sobre otra con diferentes estadísticos en función de las variables y de la prueba, en este caso, se ha calculado la $d$ de cohen y la eta cuadrado $\eta 2$ y la $V$ de cramer.

\section{Resultados}

En relación al objetivo del estudio, establecer las motivaciones del estudiantado de la UAL hacia la práctica de actividad físico- deportiva de tiempo libre, se han obtenido los resultados que se muestran a continuación.

El estudio de la motivación se subdivide en dos grandes apartados, por un lado, analizar los motivos por los que realizan la práctica de actividad físicodeportiva en ofertas de la Universidad o si lo hacen por su cuenta, los motivos de abandono de la práctica de actividad física o por los que nunca han practicado. Por otro, se evaluaban los motivos por los que se practican o han practicado deportes náuticos.

Dentro de este objetivo, se ha incluido un subobjetivo que trata de aclarar cuáles son los motivos principales por los que practica actividad físico-deportiva de forma libre y/o en otras ofertas.

Los estudiantes, tal y como se aprecia en la Figura 1, como primer motivo señalan en el 25,1\% de los casos que la causa obedece a la flexibilidad horaria, adecuada a la disponibilidad 
de su tiempo libre. En segundo lugar precisaron que optaban por ello, debido a la cercanía a su domicilio en un 23,4\% de las ocasiones. En tercero, en un 17,6\% de los casos, prevaleció el hecho de asistir con amigos/as o familia. Seguido en cuarto lugar con menos de diez unidades porcentuales menos los motivos siguientes: por pertenecer a un equipo federado, club o asociación, por cuestiones económicas y porque las instalaciones son más adecuadas $(8,1 \%$, $7,4 \%$ y $5,8 \%$ respectivamente).

Como segundo motivo de practicar por su cuenta y/o en otras ofertas, entre este grupo, vuelve a aparecer la flexibilidad horaria en $22,1 \%$ de las ocasiones, seguida a dos puntos de la cercanía en el domicilio como motivos más destacados.

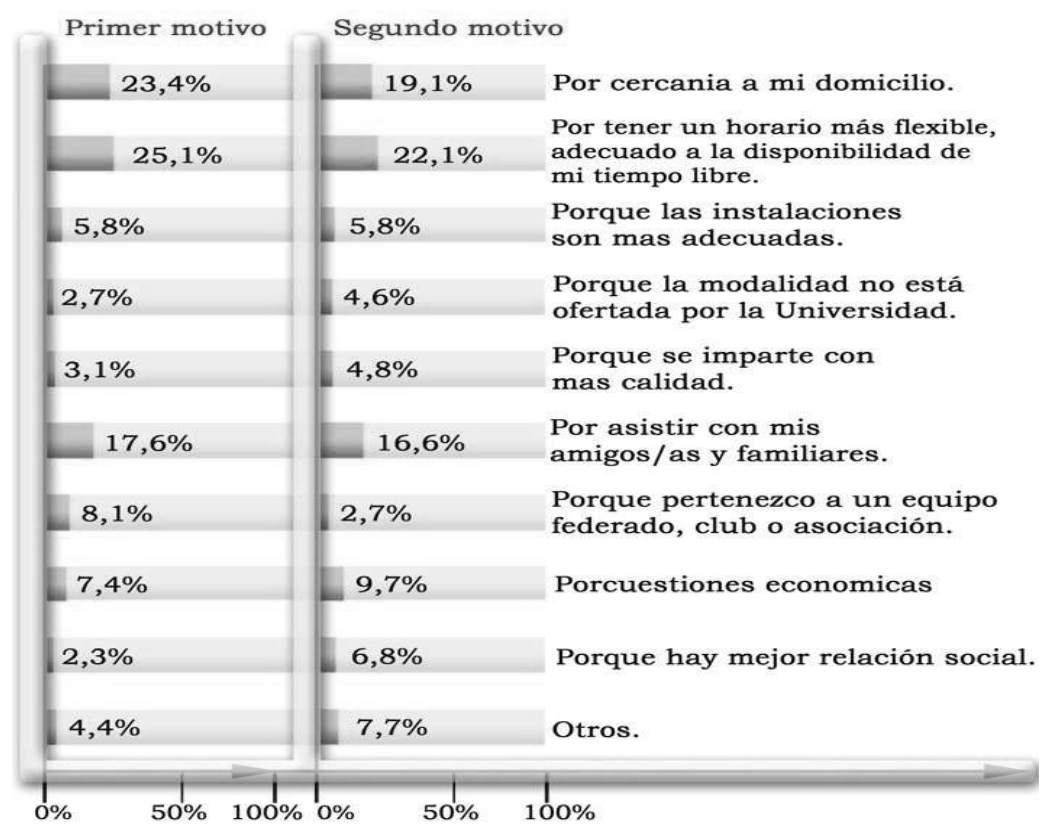

Figura1. Motivos de práctica de actividad físico-deportiva libre.

Únicamente se han encontrado diferencias significativas $(p<.05)$ en función del sexo en relación a este objetivo. De esta forma entre los tres primeros motivos señalados con mayor porcentaje, para el primer motivo se indica: la flexibilidad horaria, adecuada a la disponibilidad de su tiempo libre, no existen diferencias entre mujeres y varones. El argumento debido a la cercanía a su domicilio es usado por las mujeres en doce unidades porcentuales más que los varones. Por último, el hecho de asistir con amigos o familia es alegado más por los varones, en siete unidades porcentuales. Con respecto a los citados como segundo motivo aparecen: la flexibilidad horaria, adecuada a la disponibilidad de su tiempo libre, las mujeres se adelantan en once unidades porcentuales a los varones. Con respecto al motivo por cercanía a su domicilio se diferencian en tan sólo $0,8 \%$.Y en la preferencia de las relaciones sociales, asistir con amigos/as o familia, los varones vuelven a superar a las mujeres en cinco unidades porcentuales.

Otro subobjetivo dentro de este objetivo, pretende determinar cuáles son los motivos por los que se practican o han practicado deportes náuticos.

Como primer motivo los estudiantes de este grupo señalan su preferencia $(46,4 \%)$ por 
meras inclinaciones personales, o dicho de manera más coloquial, simplemente porque les gustan. A continuación aparece un vivo interés por estar en contacto con el medio natural y acuático (18\%). En tercer lugar, en 9,4\% de las ocasiones se ve en ellos una forma óptima de divertirse y de pasar el tiempo libre, seguido por realizarlos con un grupo de amigos (8,6\%).Con menos de cinco unidades porcentuales aparecen otros motivos como se puede apreciar en la Figura 2.

El segundo motivo sigue una orientación equivalente al del motivo anterior entre los más señalados. Un 20,5\% alude a la diversión y a pasar el tiempo libre, seguido en el $14 \%$ de las ocasiones por las meras preferencias personales. En tercer lugar se indica que es por conocer y practicar nuevos deportes $(11,5 \%)$ y así sucesivamente tal y como se puede apreciar en la Figura 2.

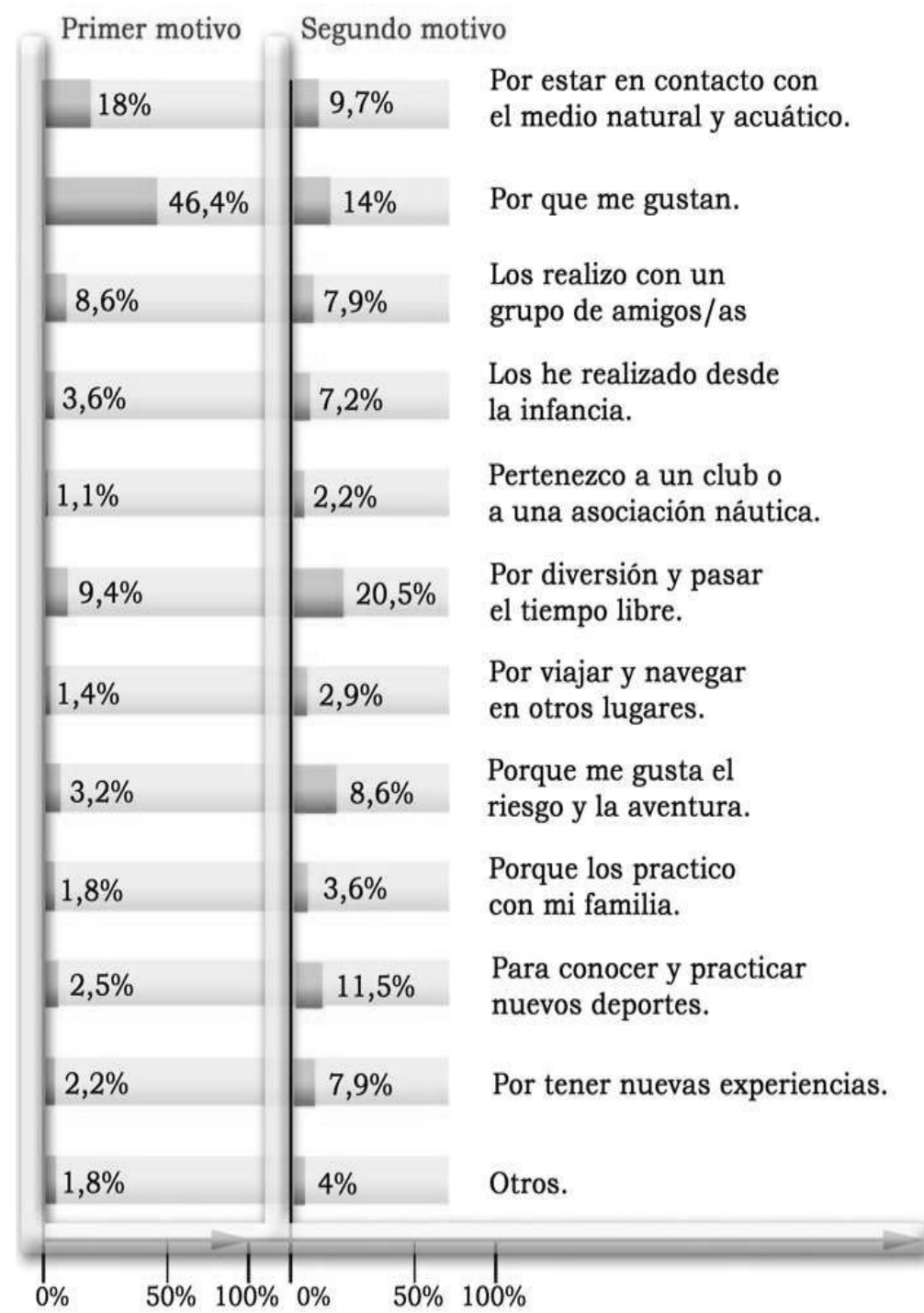

Figura 2. Motivos por los que el estudiante practica o ha practicado deportes náuticos.

Centrándonos en las cuatro causas principales como primer motivo, expuestas anteriormente y siguiendo además el mismo orden se apunta: por preferencia personal, por estar en contacto con el medio natural y acuático, por diversión y pasar el tiempo libre y por estar 
con un grupo de amigos. Esas actitudes hacia los deportes náuticos sí están, como se acaba de señalar, relacionadas desde el punto de vista generacional.

Otros motivos indican la preferencia personal, "porque me gusta”, corresponde a los más jóvenes (primer grupo de edad de 17 a 24 años) y a los más mayores (más de 40), por encima de los otros grupos de edad, siendo además la preferencia mayoritariamente indicada (49\% y $62.5 \%$ respectivamente). El interés por estar en contacto con el medio natural y acuático, en cambio, se distribuye en porcentajes similares en los cuatro grupos de edad (18\%, 20\%,16.7\% y $12.5 \%$ en cada grupo de edad), no existiendo diferencias estadísticamente significativas entre los diferentes subgrupos $(F(1)=1,390 ; p<.239, \eta 2=0.03)$. A continuación se decantan por la diversión y pasar el tiempo libre que conlleva la práctica de deportes náuticos, los más jóvenes (10\% los de17 a 24 años y $8 \%$ los de 25 a 30 años). Por último el cuarto motivo, porque lo realiza con un grupo de amigos, es predominante igualmente entre los diferentes grupos de edad $(7.1 \%$ de diecisiete a veinticuatro años, $20 \%$ de veinticinco a treinta años, $16.7 \%$ de treinta y uno a cuarenta y $12.5 \%$ más de cuarenta). Con respecto a la clase social a la que pertenece su familia cabe destacar que quienes dicen pertenecer a una situación social baja y alta se decantan en su mayoría por el motivo de estar en contacto con el medio natural y acuático (37.5\% y el $100 \%$ de cada uno de ellos). Sin embargo tanto los de clase media baja, media y media alta los realizan porque les gustan (38.5\%,50\% y 43.9\% respectivamente).No obstante, se debe resaltar que no existen diferencias estadísticamente significativas en función de la clase social de la persona $(F(4)=0,857 ; p<.490, \eta 2=0.008)$.

En relación al siguiente subobjetivo, se trata de determinar cuáles son los motivos de abandono de la práctica deportiva. Entre las diferentes razones planteadas para explicar dicho abandono la más señalada y claramente distanciada del resto, es la falta de tiempo porque el estudio, o el trabajo, le exigían demasiado $(90,8 \%)$.

En segundo lugar, un 48,7\% sostiene no continuar haciendo prácticas físico-deportivas en la actualidad por salir demasiado cansado del trabajo o muy tarde, seguido por un $47,8 \%$ que reconoce en ello simplemente pereza y desgana. A continuación con siete unidades porcentuales menos alegan c o m o m o t i vo la distancia de la instalación a su domicilio.

Con más de 20 unidades porcentuales son señalados los siguientes motivos: porque le gustaban más otras cosas, por otras razones y por motivos económicos $(22,8 \%, 22,5 \%$ y $21,5 \%)$.

A continuación con el mismo porcentaje, aluden por falta de apoyo y estímulo y porque sus amistades no practicaban $(17,4 \%)$.Seguidos por motivos relacionados con la capacidad física, por lesiones(15,2\%) y por salud $(10,4 \%)$. Con menos de diez unidades porcentuales son señaladas las causas que se especifican en la Figura 3. 


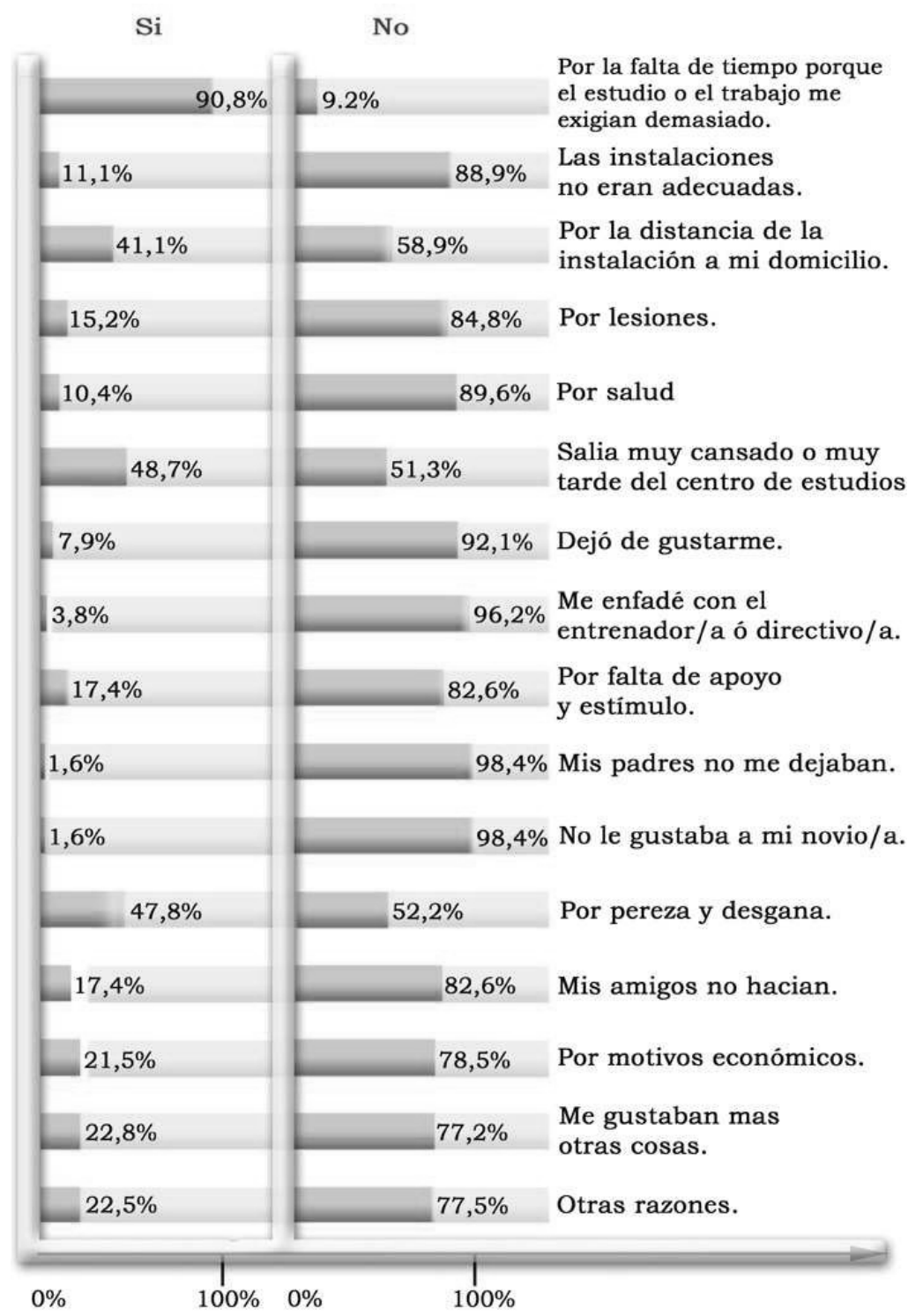

Figura 3. Causas del abandono de práctica físico-deportiva.

Tratando de establecer una prelación jerárquica en esas motivaciones, a los estudiantes que han abandonado totalmente estas prácticas se les solicitó que organizaran, la causa principal que había influido en esa decisión. Más de la mitad de la muestra señaló en primer lugar la falta de tiempo, a causa de las exigencias laborales o de estudios (56,3\%),seguido por la pereza o simple desgana (7,9\%), para concluir con las lesiones $(6,6 \%)$ y por salud $(6 \%)$, la novedad más significativa que aparece en este nuevo listado.

La subespecificación posterior en función de la variable sexo, muestra diferencias significativas $(p<.05)$.Las mujeres señalan que sí les ha influido al abandono la distancia de la instalación a su domicilio, en más de doce unidades porcentuales más que los varones $\left(\chi^{2}(1, N\right.$ $=316)=4,568, p=.033 ; V=.120$ ). Igualmente, en la causa porque salía muy cansado o muy tarde del centro de estudios, existen 11 unidades porcentuales de diferencia entre ambos, aunque en este caso la diferencia no es significativa $p=.066$. 
Por el contrario, es más llamativo el 7.1\% de alumnos que manifiestan preferir otras diversiones a la actividad físico-deportiva (me gustaban más otras cosas). Hay una tendencia marcada por el factor sexo en la distribución de estos resultados, situándose los varones en dieciséis unidades porcentuales más que las mujeres $\left(\chi^{2}(1, N=316)=10,409, p=.001 ; V=\right.$ .181).

Como último subobjetivo de este apartado, se ha tratado de aclarar cuáles son los motivos por los que nunca han practicado actividad físico-deportiva.

Las razones del por qué nunca han practicado actividad físico-deportiva de tiempo libre han sido: la falta de tiempo (72,9\%); salir muy cansado del trabajo o del estudio (65,3\%); tener pereza y desgana (62,3\%); porque no hay instalaciones cerca y/o no son adecuadas (40,3\%); porque no se lo enseñaron en el centro de estudios (31,3\%); no le gusta la actividad física (25\%); no le ve utilidad y/o no lo ve beneficios $(11,8 \%)$ y con menor porcentaje los que aluden motivos de salud(6,3\%). Hay que reflejar que un $42 \%$ señala además otras razones por las que nunca ha practicado. Estas últimas motivaciones, un tanto dispersas, en todo caso han quedado resumidas en la Figura 4.

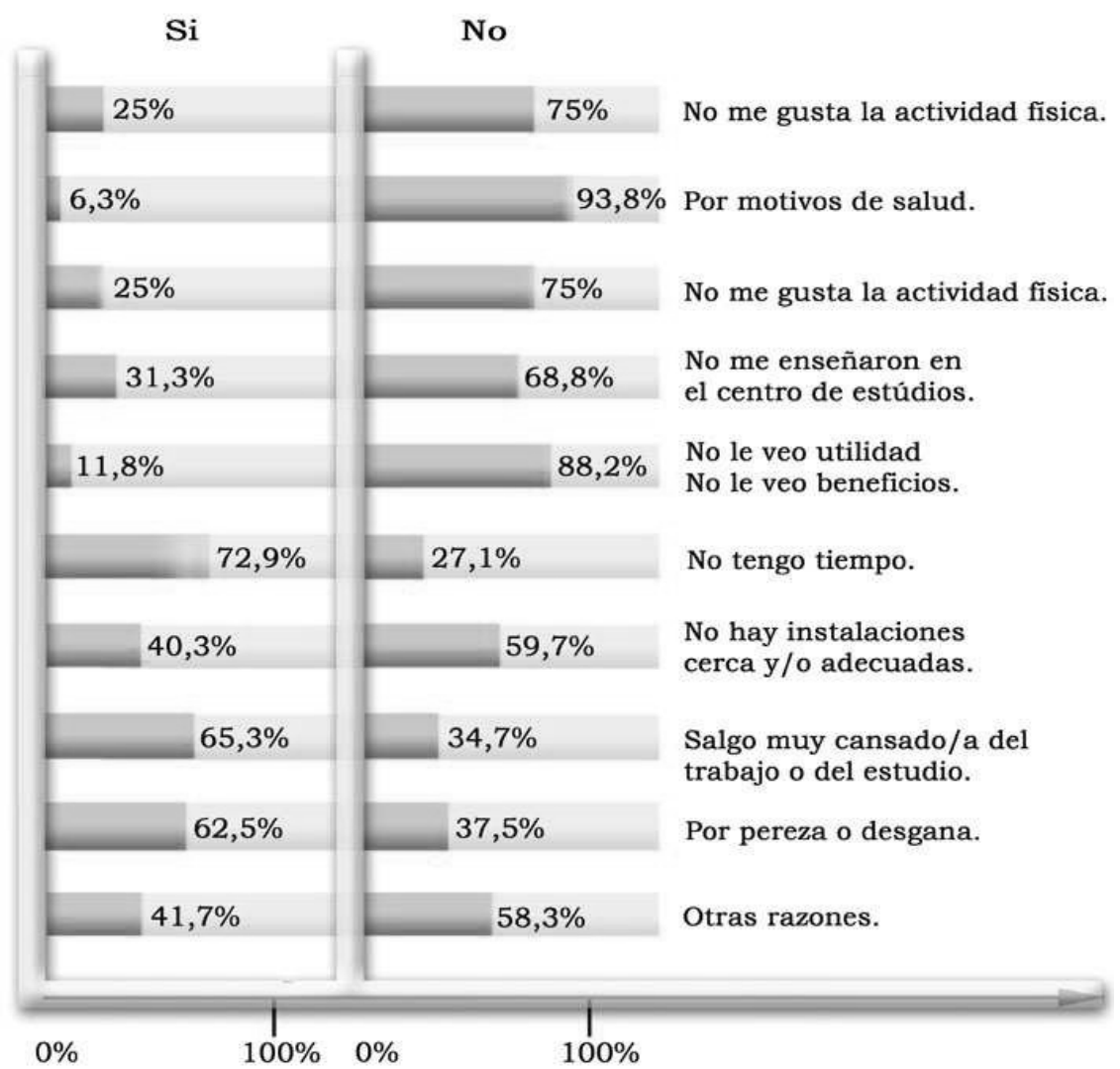

Figura 4. Motivos por los que el estudiante nunca ha practicado actividades físico-deportivas

El último objetivo desarrollado ha sido determinar qué deportes náuticos y actividades náuticas-recreativas de tiempo libre son practicadas por el alumnado de la UAL. 
Entre los estudiantes de la Universidad de Almería que practicaban actividades físicodeportivas en el momento de acometer el estudio, más de la mitad decía practicar, o haber practicado anteriormente, deportes náuticos o actividades náutico- recreativas (50,5\%).En términos absolutos, confrontando esta población con el conjunto de los estudiantes almerienses, este grupo supone un $27,5 \%$ del total de ese alumnado, cifra verdaderamente considerable que, en todo caso, parece no traducir se en una demanda efectiva de la oferta realizada por la UAL. Se diría, por tanto, que existe un potencial favorable a la náutica, no siempre aprovechado al completo.

Entre los estudiantes que tienen su domicilio familiar habitual en la provincia de Almería y que han tenido experiencias con actividades o deportes náuticos superan en veinte unidades porcentuales los de municipio de costa a los de municipio de interior. La influencia del medio cercano a su domicilio y un mayor número de ofertas puede que conlleve este mayor porcentaje en la práctica de actividades y deportes náuticos.

Dentro de este objetivo, se ha creado un subobjetivo 3.1 que trata de determinar cuáles son las modalidades de deportes náuticos o actividades náuticas-recreativas que practican o han practicado los estudiantes de la UAL.

Las modalidades de deportes náuticos o actividades náutico- recreativas, más practicadas son las que se pueden apreciar en la Figura 5, siendo el más practicado el piragüismo con un $20,2 \%$. El resto de modalidades se distribuyen con porcentajes menores al 12\%.

\section{Figura 5}

Aclarando el subobjetivo 3.2., se ha tratado de determinar cuáles son los lugares donde los estudiantes practican o han practicado deportes náuticos o actividades náuticas- recreativas.

El 34,9\% señala que practica, o ha practicado, en las playas del municipio de Almería, seguido de los que señalan la costa de Levante (24,5\%).A continuación se especifica el Centro de Actividades Náuticas(19,4\%).Posteriormente se sitúa con un 17,4\% la costa de poniente. En último lugar destaca que el centro de actividades náuticas de Almería con un 16,5\%.

\section{Discusión y conclusiones}

Respecto a las causas que aproximan a los individuos al mundo físico-deportivo, existe una díada básica que concita causas internas y externas (Dosil, 2004). Estas, básicamente, apuntan al gusto por la competición en el primer supuesto. En el segundo se inclinan por intereses más de tipo hedonístico. A ello habría de agregarse un tercer factor, vinculado en esta ocasión con el relax, así como una mayor consciencia acerca de la positiva repercusión de estas actividades sobre la salud, en general. Por último, se ha señalado también su capacidad para fomentar las relaciones sociales (Castillo-Fernández et al., 2000).

En el terreno empírico, la bibliografía al uso ha corroborado esas tendencias (Castillo, 2011; García-Ferrando, 2001 y 2005; Otero, 2004 y 2009; Puig, Villanova, Inglés y Mayo, 2009). En esos trabajos se consigna un elenco bastante regular de motivaciones, con independencia de la comunidad concreta que se observe.

En ese sentido, se tiende a realizar deporte para mantenerse en forma, por la diversión que conlleva aparejada su práctica, que en ocasiones es interpretada como una forma de (c) Psy, Soc, \& Educ, 2016, Vol. 8(3) 
evasión.

Esos motivos comunes en general a todas las poblaciones, reciben una bastante acusada estratificación en algunos puntos, sobre todo procedente de la intervención del factor sexo. Así, las mujeres suelen ser más sensibles a los aspectos vinculados con la salud mientras que entre los hombres predomina el mero interés por el ejercicio. A modo de ilustración de lo comentando, en Cataluña, conforme a los datos de Puig et al., (2009)entre las tendencias más claramente femeninas serían las relacionadas con la salud (16\% por encima de los hombres) y el mantenimiento de la buena figura (12\% por encima de los hombres). Los hombres, en cambio, predominan en subrayar el componente de relaciones sociales(14 puntos por encima de las mujeres), junto con el aspecto festivo del deporte (18 puntos por encima de las mujeres). De todas formas, es probable que nos hallemos ante una transformación relativamente profunda de las causas que conducen a la práctica deportiva. Conforme a los datos de Fraile y De Diego (2006) tomados a la población adolescente de España, Italia, Francia y Portugal, estaríamos asistiendo a un vínculo relativamente fuerte entre actividad deportiva y mejora de la salud, siguiendo una línea apuntada por Piéron, Telama, Almond y Carreiro, (1999), que ya incluía la salud junto con otras valoraciones más proclives al componente festivo de la actividad deportiva. Esos mismos parámetros se mantienen en el contexto almeriense, subrayándose sobre todo los aspectos vinculados a la forma física y la salud. Finalmente, en relación al género, se ha de notar una menor motivación por parte de la mujer hacia la práctica de la actividad física, en línea con los resultados de Cecchini, Echevarría y Méndez-Giménez (2003, citado en Méndez-Giménez, Fernández-Río, Cecchini y González, 2013).

Águila (2005) especifica que existen también factores subjetivos asociados al ocio, tales como la diversión y por la libertad que conllevan estos deportes. El desinterés, en esta ocasión, obedece a causas relativamente previsibles, como la pereza, la desgana o el no haber iniciado estas actividades nunca y, en consecuencia, carecer de lo que podríamos denominar tradición personal. En sentido amplio, por tanto, se diría que el estilo de vida eminentemente sedentario es el gran causante de este comportamiento. En todo caso, hay algunos datos singulares al respecto de todo lo que estamos comentando García-Ferrando (2001) señalaba que se había registrado un descenso sintomático entre quienes argumentaban carecer de tiempo, circunstancia que se repetía en Otero (2004), respecto de datos anteriores obtenidos en 1999. Ello se veía compensado con el incremento de quienes reconocían desagrado ante el deporte, cuya cifra había aumentado en contraposición a las dos anteriores. En Hernández, García y Oña (2001) aparecen algunas causas más explícitas, y probablemente también más explicativas, entre las que sobresale la incompatibilidad con el trabajo y/o el estudio. En ese sentido, la actividad físico-deportivo “exige demasiado” y llega un momento en que resulta incompatible con otras que deben desarrollarlos individuos en su cotidianidad. A ellos hay que agregar, el cansancio provocado por el trabajo o el estudio, y la siempre presente "falta de tiempo"; todos ellos constatados en la muestra que aquí manejamos.

Existe, de todas formas, un grupo singular, como es el formado por aquel sector de la población que practicó, en otro tiempo, alguna clase de actividad físico-deportiva pero que, por un conjunto diverso de razones, ha procedido a abandonarla. Conforme han señalado diferentes autores (Balaguer, Pastor y Moreno, 1999; Blasco, Capdevila, Pintanel, Valiente y Cruz, 1996; Castillo, 2011; García Ferrando, 2001, 2005; Gómez, 2005; Otero, 2004, 2009; Puig et al., 2009; Ulla y Pérez, 2009) existirían dos momentos de fractura con la práctica físico-deportiva: 
el primero se situaría en la transición de la enseñanza secundaria obligatoria al bachillerato; el segundo, al pasar de este a la universidad. En ambos, se combinarían, tanto las transformaciones psicológicas de la adolescencia, como por otra parte la modificación sustancial de los hábitos de vida de esta población, al tener que enfrentarse a un nuevo escenario educativo. Ello se traduce, como es lógico, en argumentaciones vinculadas con la falta de tiempo, la pereza o la desgana, tal y como registran Hernández-Rodríguez, García y Oña (2001), Ruiz, García y Hernández (2001) o Gómez (2005). Se registra, asimismo, la intención de recuperar la actividad físico- deportiva que se ha perdido en un futuro más o menos mediato.

\section{Limitaciones y prospectiva}

Entre las limitaciones que presenta el estudio, indicar que la validación de este estudio debe tener continuidad en el tiempo, la utilización de diferentes muestras y más estudios que comprueben la validez y fiabilidad de estos resultados. De igual forma, las motivaciones hacia la práctica físico-deportiva debería ser comprobada en otros contextos educativos. También, futuras investigaciones deberían comprobar los efectos del género y la edad sobre la práctica de deportes náuticos dentro de los comportamientos sobre actividad-física y deporte de tiempo libre y el desarrollo estrategias comunicativas que favorezcan el clima de apoyo hacia los deportes náuticos.

\section{Referencias}

Almagro, B. J., Sáenz-López, P. y Moreno-Murcia, J. A. (2012). Perfiles motivacionales de deportistas adolescentes españoles. Revista de Psicología del Deporte, 21(2), 223-231.

Alonso, D. y García, J. L. (2010). Motivación hacia la práctica físico-deportiva de universitarios gallegos. Revista de Investigación en Educación, 8, 128-138.

Águila, C.(2005). Ocio, jóvenes y posmodernidad. Almería: Universidad de Almería.

Ames, C. (1995). Metas de ejecución, clima motivacional y procesos motivacionales. En G. Roberts (Ed.), Motivación para el deporte y el ejercicio. Bilbao: Desclée

Balaguer, I., Pastor, Y. y Moreno,Y. (1999). Algunas características de los estilos de vida de los adolescentes en la Comunidad Valenciana. Revista Valenciana d'Estudis Autonómics,26, 33-56.

Blasco, T., Capdevila, L., Pintanel, N., Valiente, L. y Cruz, J. (1996). Educación de los patrones de actividad física en estudiantes universitarios. Revista de Psicología del Deporte, 9(10), 51-63.

Castillo, I. Balanguer, I. y Duda, J. J. (2000). Las orientaciones de meta y los motivos de práctica deportiva en los jóvenes deportistas valencianos escolarizados. Revista de Psicología del Deporte, 9(1-2), 37-50.

Castillo, E.y Jiménez, F.J.(2011). Hábitos de práctica de actividad física del alumnado de la universidad de Huelva. Revista Internacional de Medicina y Ciencias de la Actividad Física y el Deporte, 10(41), 127-144.

Centro de Investigaciones Sociológicas. (2005). Los hábitos deportivos de los españoles (III). Boletín de datos de opinión. Estudio CIS2599. Recuperado de http://www.cis.es/cis/ 
opencms/ES/index.html.

Deci, E., y Ryan, R. (2000). The «what» and «why» of goal pursuits: Human needs and the self- determination of behaviour. Psychological Inquiry, 11, 227-268.

Delgado, M. y Tercedor, P. (2002). Estrategias de intervención en Educación para la salud desde la Educación Física. INDE: Barcelona.

Dosil, J. (2004). Psicología de la Actividad Física y del Deporte. Madrid: McGraw-Hill.

Duda, J., y Nicholls J. L. (1992). Dimensions of Achievement motivation in school-work and sport. Journal of Educational Psychology, 84, 290-299.

García Ferrando, M. (2001). Los españoles y el deporte: prácticas y comportamientos en la última década del siglo XX. Encuesta sobre hábitos deportivos de los españoles. Madrid: Ministerio de Educación, Cultura y Deporte.

García Ferrando, M. (2005). Postmodernidad y Deporte: Entre la Individualización y la masificación. Encuesta sobre hábitos deportivos de los españoles 2005. Madrid: Consejo Superior de Deportes.

García Ferrando, M., y Llopis, R. (2010). Ideal democrático y bienestar personal: Encuesta sobre los hábitos deportivos en España 2010. Madrid: Consejo Superior de Deportes.

Gómez López, M.(2005). La actividad físico-deportiva en los almerienses de Educación Secundaria Post obligatoria y en la Universidad de Almería. Educación físicodeportivas de su alumnado. Almería: Servicio de Publicaciones de la Universidad de Almería.

Fraile, A. y De Diego, R. (2006). Motivaciones de los escolares europeos para la práctica del deporte. Un estudio realizado en España, Italia, Francia y Portugal. Revista Internacional de Sociología, 44, 85-109.

Hernández, A.I., García, E. y Oña, A. (2001). Conocimiento y valoración del servicio de deportes de la Universidad de Almería. En P. Martos, J. L., Paniza y B. M. Latiesa (2001), Vol. I, 337-390.

Martínez, A., Hellín, P., Pavón, A. y Moreno, J.A. (2010). Motivos de práctica físico-deportiva. En J.A. Moreno y E. Cervelló (Coords.), Motivación en la actividad física y el deporte. Sevilla: Wanceulen.

Méndez-Giménez, A. y Fernández-Río, J., Cecchini, J. A. y González, C. (2013). Perfiles motivacionales y sus consecuencias en educación física. Un estudio complementario de metas de logro 2x2 y autodeterminación. Revista de Psicología del Deporte, 22(1), 2938.

Nicholls, J. (1984). Achievement motivation: conceptions of ability, subjective experience, task choice and performance. Psychological Review, 91, 328-346

Otero, J.M. (2004). Hábitos y actitudes de los andaluces ante el deporte. Cádiz: Consejería de Turismo y Deporte. Observatorio del Deporte Andaluz.

Otero, J.M. (2009). Hábitos y actitudes de la población andaluza ante el deporte. Sevilla: Empresa Pública.

Pavón, A., y Moreno, J. A. (2006). Diferencias por edad en el análisis de la práctica físicodeportiva de los universitarios. Cuadernos de Psicología del Deporte, 6(1), 53-67.

Pavón, A., y Moreno, J. A. (2008). Actitud de los universitarios ante la práctica físicodeportiva. Revista de Psicología del Deporte, 17(1), 7-23.

Pavón, A., Moreno, J. A., Gutiérrez, M. y Sicilia, A. (2003). La práctica físico-deportiva en la 
Universidad. Revista de Psicología del Deporte, 12(1): 23-31.

Pierón, M. Telama, R. Almond. L. y Carreiro, F. (1999). Estilo de vida de jóvenes europeos: un estudio comparativo. Revista de Educación Física, 76, 5-13.

Puig, N., Villanova, A., Inglés, E. y Mayo, D. (2009). Hábitses por tiusa Catalunya. Barcelona: Generalitat de Catalunya.

Ruiz, F., García, E. y Hernández, A. I. (2001). El interés por la práctica de actividad físicodeportiva de tiempo libre del alumnado de la Universidad de Almería. Un estudio longitudinal. Apunts. Educación Física y Deportes, 63, 86-92.

Roberts, G., y Treasure, D. (1995): Motivational determinants of achievement of children in sport. Revista de Psicología del Deporte, 7-8, 123-134.

Ryan, R., y Deci, E. (2007). Self determination theory and the facilitation of intrinsic motivation, social development and well-being. American Psychologist, 55, 68-78.

Ulla, S. y Pérez, A. (2009). Los hábitos de salud de los estudiantes universitarios de Castilla la Mancha. Cuenca: Universidad de Castilla la Mancha.

Wigfield, A., y Eccles, J.S. (2000). Expectancy-value theory of theory of achievement motivation. Contemporary Educational Psychology, 25, 68-81. 